\title{
Highly Dispersed, Adhesive Carbon Nanotubes Ink for Strain and Pressure Sensors
}

\section{Supporting Information}

Qiuyan Duan, Bijian Lan ${ }^{\mathrm{b}}$, Yinxiang Lva,c*

a Department of Materials Science, Fudan University, Shanghai 200433, China

b Taicang Biqi New Materials Research and Development Inc., Jiangsu 215431, China

c Yiwu Research Institute of Fudan University, Zhejiang 322000, China

*Corresponding Author E-mail: yxlu@fudan.edu.cn. Tel. \& fax: +86 2155665059. 


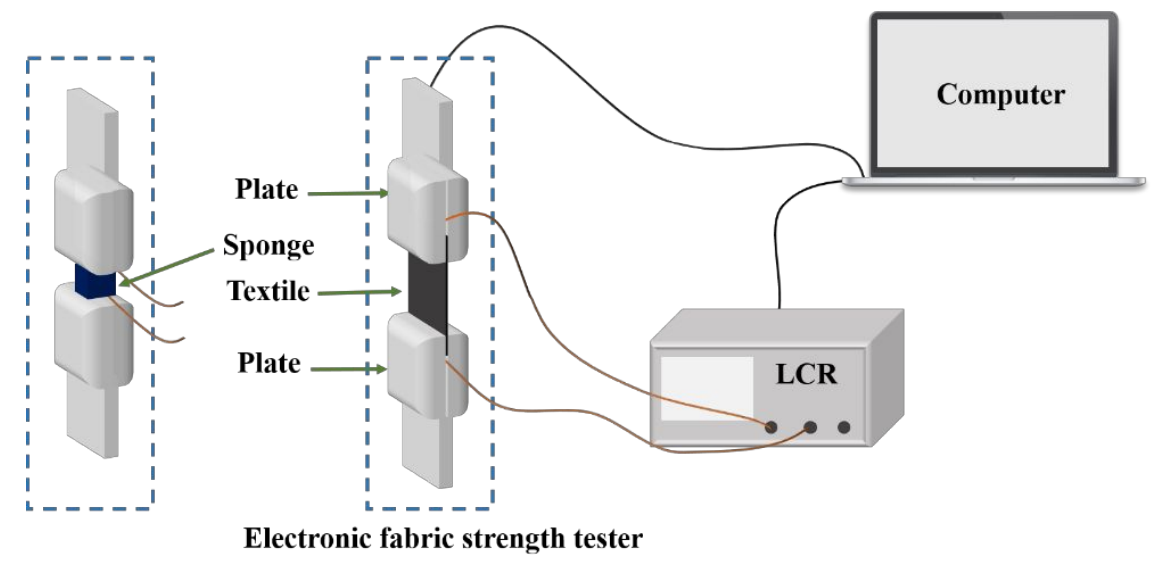

Figure S1. Experimental setup for characterization of sensing performance.

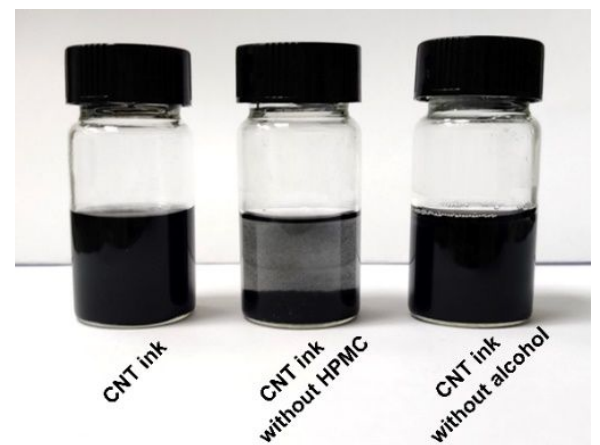

Figure S2. Comparation of CNT ink and its control groups. 

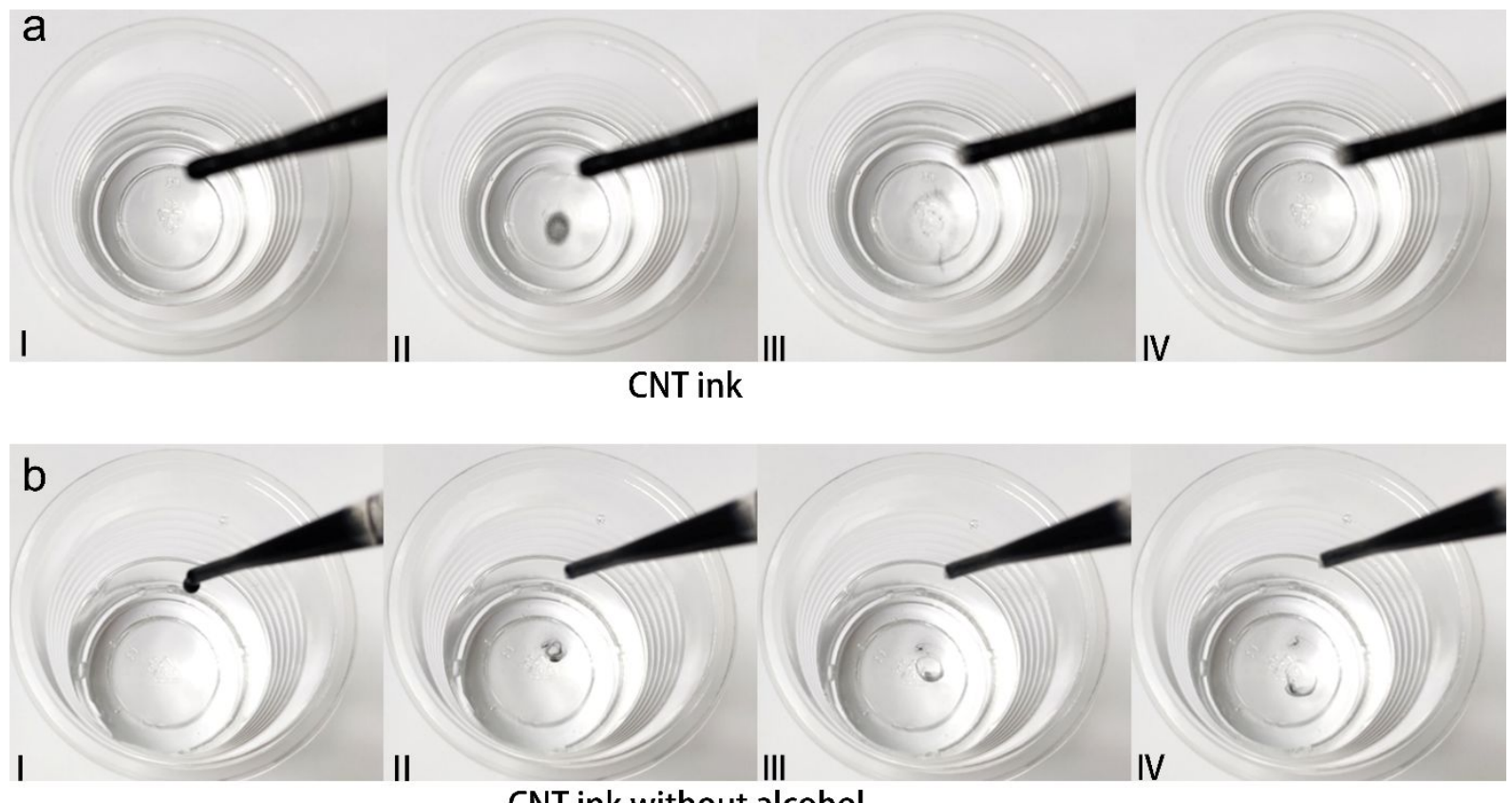

CNT ink without alcohol

Figure S3. The evolution process of a) CNT ink droplets and b) CNT ink without alcohol droplets added into water during 2 seconds.
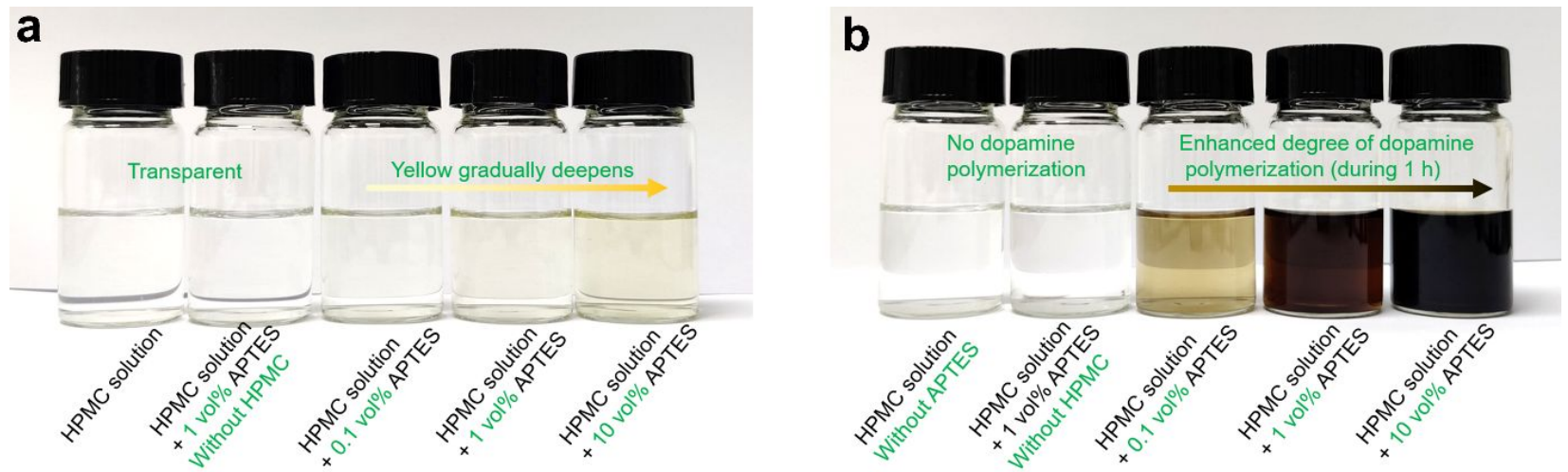

Figure S4. a) Color changes after HPMC reacted with different contents of APTES at $110^{\circ} \mathrm{C} . \mathrm{b}$ ) Color changes when dopamine polymerization in HPMC with different concentrations of APTES in half an hour. 


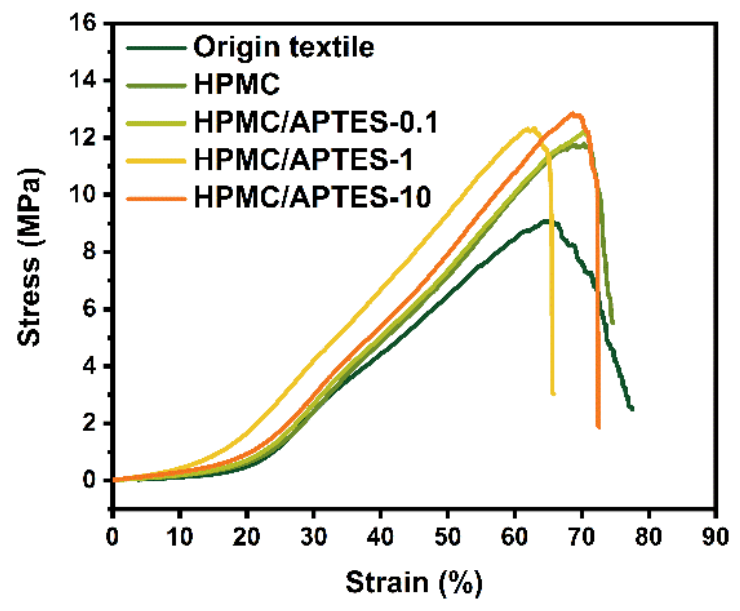

Figure S5. Tensile strength test of acetate rayons coated with HPMC and its derivatives respectively.

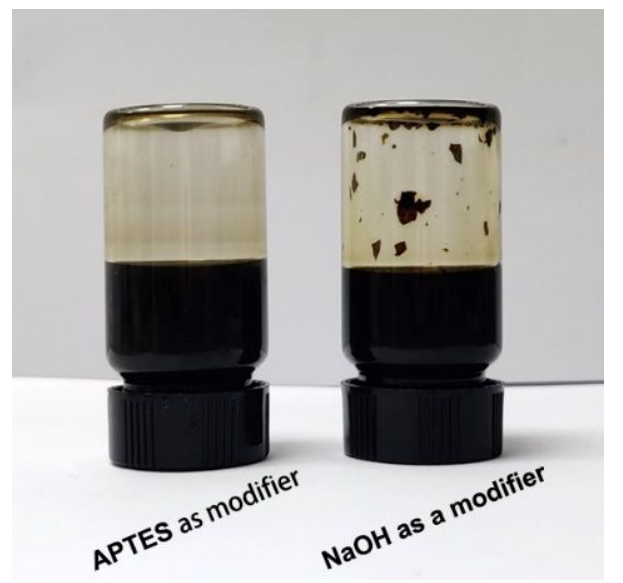

Figure S6. Comparation of polydopamine standing for one month when APTES and $\mathrm{NaOH}$ were used as induce agents respectively. 


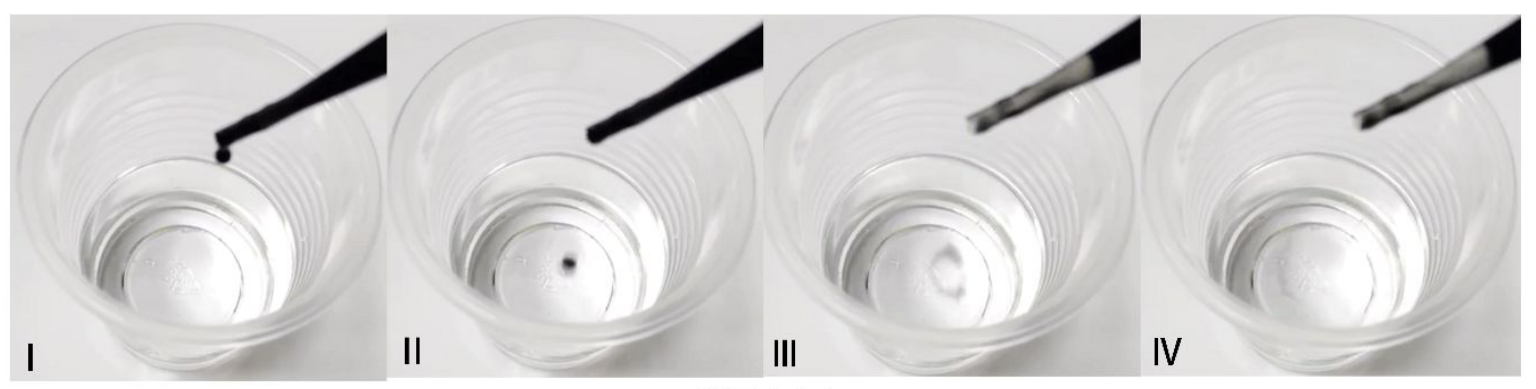

CNT-A ink

Figure S7. The evolution process of CNT-A ink droplets added into water during 2 seconds.

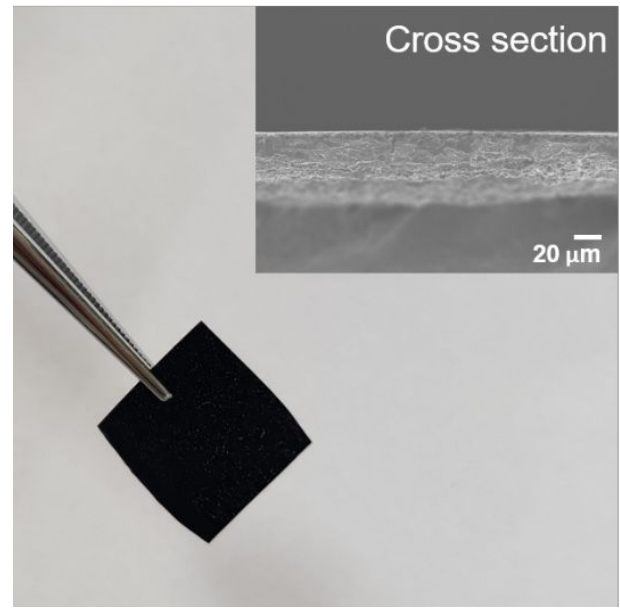

Figure S8. Photograph of CNT-A film prepared via drop casting. Inset shows SEM image of crosssection of CNT-A film. 

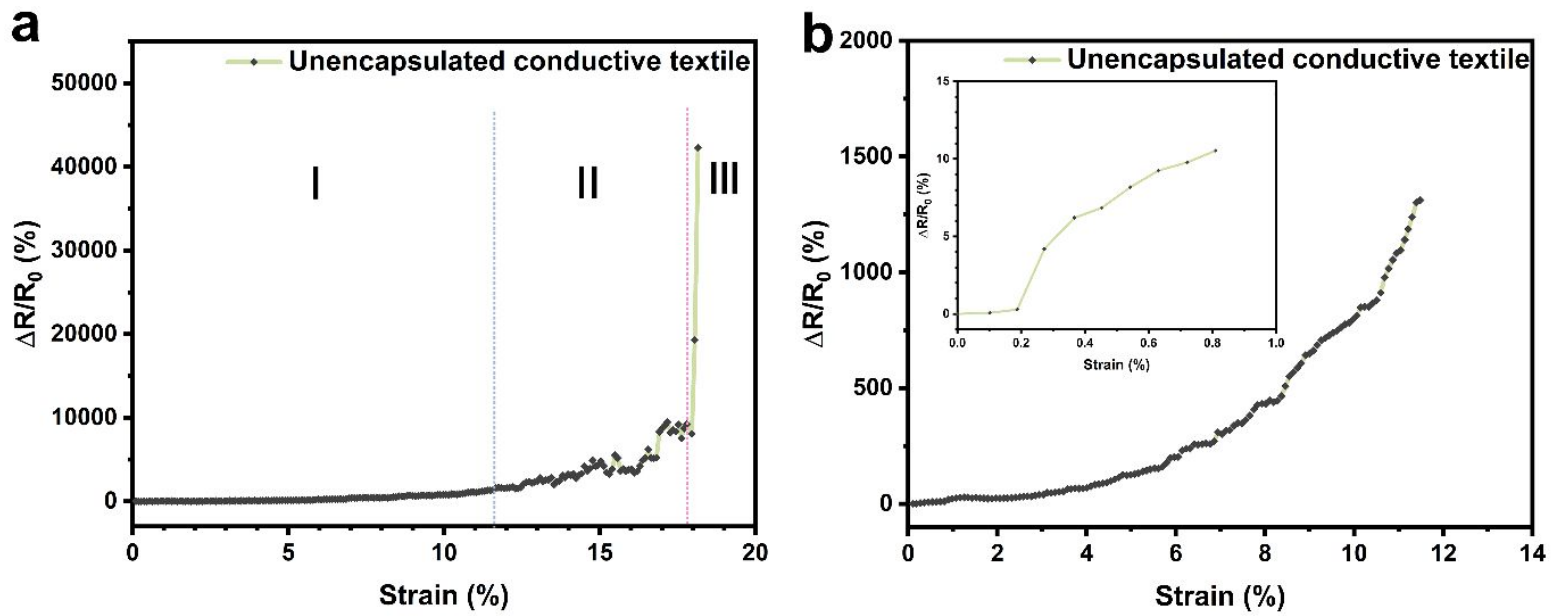

Figure S9. a) The relationship between relative resistance changes and the applied strain of printed conductive textile. b) The enlarged view of part $I$ in a). The inset is the relative change in resistance under tiny strain at the beginning.
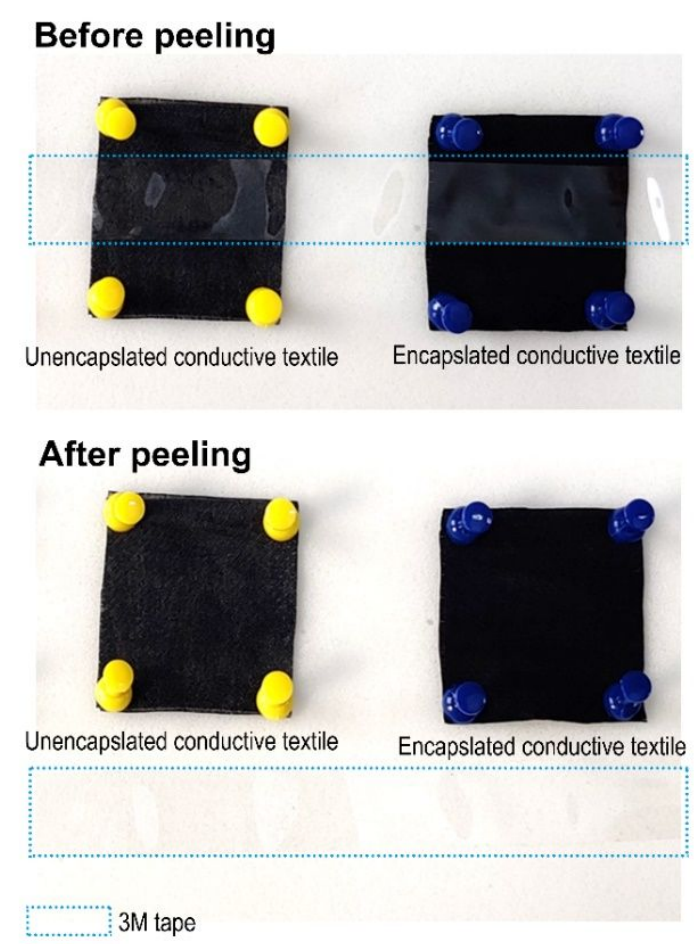

Figure S10. 3M tape test of the unencapsulated and encapsulated conductive textile. 
a

Tapping

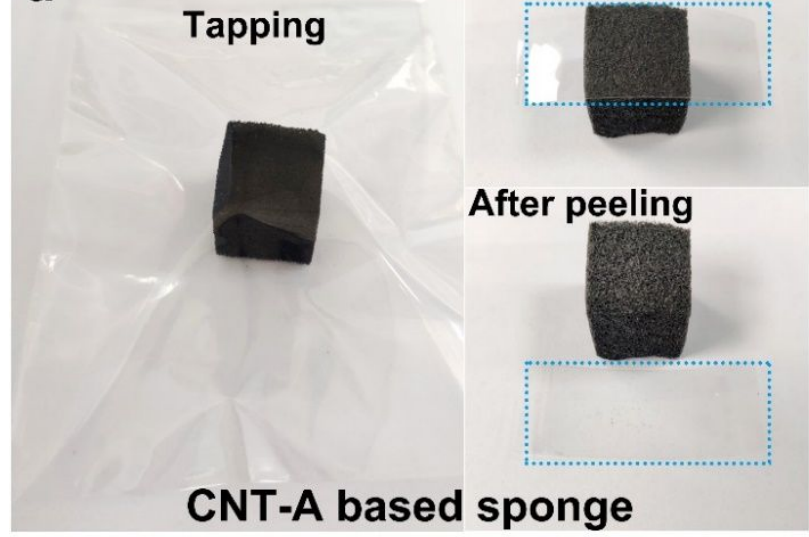

$3 \mathrm{M}$ tape b

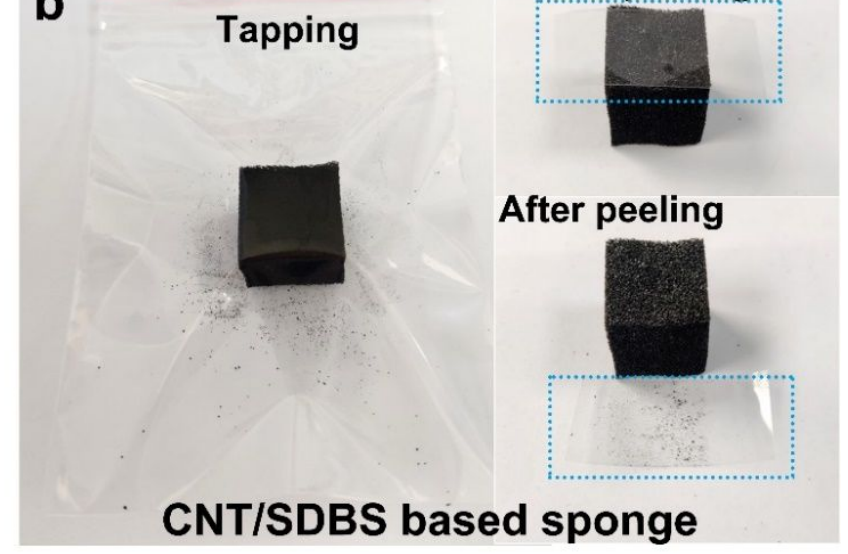

Figure S11. Adhesion test of CNT-A and CNT/SDBS on sponges by tapping and 3M tape peeling. 